\title{
A Game-Theoretic Price Determination Algorithm for Utility Companies Serving a Community in Smart Grid
}

\author{
Tiansong Cui, Yanzhi Wang, Siyu Yue, Shahin Nazarian, and Massoud Pedram \\ University of Southern California \\ Department of Electrical Engineering \\ Los Angeles CA USA \\ \{tcui, yanzhiwa, siyuyue, shahin, pedram $\} @$ usc.edu
}

\begin{abstract}
Distributed power network is the major trend of future smart grid, which contains multiple non-cooperative utility companies who have incentives to maximize their own profits. The energy price competition forms an $\boldsymbol{n}$-person game among utility companies where one's price strategy will affect the payoffs of others. More interestingly, the use of dynamic energy pricing schemes incentivizes homeowners to consume electricity more prudently in order to minimize their electric bill. In this paper, two models of price determination are introduced for utility companies under different assumptions. In the first model, a Nash equilibrium solution is presented and the uniqueness of Nash equilibrium point is proved. The second model accounts for more sophisticated factors such as the cost of energy generation and the homeowner's reaction to the change of energy usage as a factor of energy price. Although it is no longer possible to prove the uniqueness of Nash equilibrium for the second model, we present a practical solution in which no utility company can increase its expected profit by adjusting the price function. Experimental results show the effectiveness of our two models both in reliability of solution and in runtime.
\end{abstract}

\section{INTRODUCTION}

The current smart grid technology is undergoing a transformation from a centralized, producer-controlled network to one that is less centralized and more consumerinteractive [1][2]. This will lead to a change of the industry's entire business model and its relationship with all stakeholders, involving and affecting utilities, regulators, energy service providers, technology and automation vendors and all consumers of electric power [1]. With the introduction of decentralized electrical network architecture, the usual practice of power network on matching supply to real-time demand will be increasingly challenging [3]. It is generally agreed that the amount of generation, transmission and distribution capacities that utility companies need to provision depends on peak demand rather than the average [4]. Consequently, the huge difference between energy consumption levels at peak usage time and off-peak hours has resulted in not only cost inefficiencies and potential brownouts and blackouts, but also environmental pollution due to over provisioning of the Power Grid and the resulting energy waste [5]. For example, the US national load factor is about $55 \%$, and only $10 \%$ of generation plants and $25 \%$ of distribution facilities are used less than 400 hours per year, i.e., $5 \%$ of the time [1].

The introduction of dynamic energy pricing gives a popular solution on shaping the demand to reduce the peak and smoothen the variation [1]-[4]. Dynamic pricing is an incentive-based scheme, which means both utility companies and energy users have an incentive to maximize their own profits or minimize their own costs. By dynamic changing of energy prices, it will be possible for customers to shift their energy consumption from peak-energy-use hours to off-peak times, thus lower their monthly electricity bills [5][6]. Proper shaping of customer's demands makes it possible for utility companies to reduce their capital expenditure by not having to add new power plants to the Grid in order to meet the customers' peak-hour demands. Dynamic energy pricing is therefore an economical scheme that will benefit both the energy consumers and the utility companies.

Although dynamic pricing is well researched, several problems still exist for the scheme to become practical. First of all, most of previous papers have focused on either profit maximization for utility companies [4] or cost minimization for customers [5][8]. However each of the utility companies and customers tend to make its decision based on the reaction of the other side. In addition, almost all the existing models are based on a centralized monopolistic electrical grid, where a single utility company supplies all the power demands of electricity consumers in a local area. But as a decentralized smart grid is the major trend of future electrical power network architecture [1], competition between different utility companies will be increasingly widespread. Although an oligopolistic energy market was introduced in [17], it is only possible for utility 
companies to optimize part of their profits from homeowners whose "threshold price" are higher than the offered price, and the profit from wining the price competition is claimed to be "unpredictable" and thus ignored in the optimization process. Failing to take into account the interactions among different utility companies, this model turned out to be oversimplified.

Due to the above reasons, a more accurate and practical model is needed to solve the profit maximization problem in an oligopolistic energy market. Based on the studies in [18], game theory has been expected to constitute a key analytical tool in the design of the future smart grid. Game theory is a formal analytical as well as conceptual framework with a set of mathematical tools enabling the study of complex interactions among independent rational players [18]. In a real-life price competition, utility companies can be considered as the rational players and tend to analyze their competitors' decisions and give their corresponding responses accordingly. In addition, it is also necessary for the utility companies to recursively adjust their price functions and finally reach a "Nash equilibrium" [12].

Considering these factors, two models of dynamic pricing are presented in this paper to solve the profit maximization problem for utility companies under an oligopolistic energy market. For both models, we assume that each energy consumer has the ability to freely select any of the existing utility companies without any additional cost, and it is the energy price function that determines the probability he/she selects a certain company. Based on this assumption, we propose game theoretic solutions for determining the hourly price of the electric energy in each utility company to maximize its total expected profit. In the first model, a Nash equilibrium solution is presented and the uniqueness of Nash equilibrium point is proved. The second model considers more sophisticated factors such as the cost of energy generation and the homeowner's reaction on the change of energy usage as a factor of energy price. Although it is no longer possible to prove the uniqueness of Nash equilibrium, we still present a practical solution to profit maximization problem in which no utility company can increase its expected profit by adjusting the price function.

The remainder of this paper is organized as follows. In the next two sections, we present our two models as well as the corresponding solutions for profit maximization of utility companies in oligopolistic market. Section IV reports the simulation results and the paper is concluded in Section V.

\section{MODEL I: IGNORING ENERGY GENERATION COST}

In this paper, a slotted time model is assumed for all models, i.e., all system cost parameters and constraints as well as scheduling decisions are provided for discrete time intervals of constant length. The scheduling epoch is thus divided into a fixed number of equal-sized time slots (in the experiment, a day is divided into 24 time slots, each with duration of 1 hour). A unified electricity bill is used throughout the paper.

We define Price function, $P[c][t]$, as the price of one unit of energy $(\mathrm{kWh})$ for each utility company $c$ at time slot $t$. The price is decided by the utility company and preannounced to homeowners. In addition, for every homeowner $h$, con $[c][h][t]$ is the total energy consumption at time $t$ if he chooses company $c$. It can be easily observed that the equation below calculates the total energy cost for a certain homeowner:

$$
\cos _{-} h[c][h]=\sum_{t} P[c][t] \cdot \operatorname{con}[c][h][t]
$$

As stated above, our ultimate goal is to solve profit maximization problem for each utility company in oligopolistic market. But in the classical economics problems, between sellers and buyers, economists always give suggestions to the sellers based on the reaction of the buyers or vice versa because although the government would like to maximize the total social welfare, we still need to consider sellers and buyers as non-cooperative and always making decisions based on their own best solution [12]. This is also the case for energy users and utility companies, which means utility companies need to analyze homeowner's reactions based on a given price function.

It has been proved in [17] that both Cournot Model and Bertrand Model fail in the future architecture of smart grid. First of all, demand response is a key element of the smart grid technologies, which means the usual practice of power networks is matching supply to demand instead of matching demand to supply. For this reason, the Cournot Model, which is based on competition on the amount of output each industry will produce, is not applicable. On the other hand, the Bertrand Competition Model, which assumes consumers always choose the product with the lowest price, also turns out to be oversimplified, because the introduction of dynamic prices makes it hard for the consumers to determine which utility company really offers a better price, and also the customers may never be totally free to switch from one energy supply to another [12]. Hence authors in [17] used a modification of the Bertrand Competition Model which is based on the threshold cost of 
each energy consumer. But it may be unrealistic to assume that homeowners will randomly choose one utility company among those who offer a lower price than their threshold cost with an equal probability.

According to the fact that electricity is a product with almost zero elasticity [12], in this paper, a modified economical demand function is used to determine the probability that each homeowner $h$ will choose a certain utility company $c(\operatorname{prob}[c][h])$

$$
\operatorname{prob}[c][h]=\frac{e^{-\operatorname{cost}_{-} h[c][h]}}{e^{- \text {cost }_{-} h[c][h]}+\sum_{i \neq c} e^{- \text {cost }_{-} h[i][h]}}
$$

which reveals that even considering the information asymmetry, energy consumers will still have a preference to choose the company who offers a cheaper price.

To start, we consider each utility company has no cost function, i.e., the expected profit from a certain homeowner (profit $[c][h]$ ) can be calculated as follows:

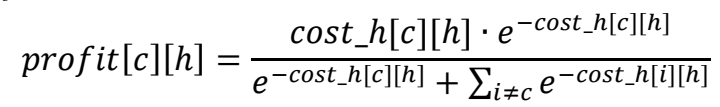

Notice that cost_h[c][h], which is an affine function of price function $P[c][t]$, is the only variable in the above equation given the price information of all other companies. If we use a single variable $x$ to represent $\operatorname{cost} h[c][h]$ and use a constant value $A$ to represent the cost summation of all other companies, the previous function can be written as:

$$
f(x)=\frac{x}{1+A \cdot e^{x}}
$$

It can be easily proved that this is a unimodal function, which means each utility company can adjust its own price function (say $x=x_{0}$ is the optimal solution) to maximize the profit. But we can also observe from the equation that the optimal price function of each company is determined by the constant $A$, which is a function of prices of other companies. On the other hand, the price decision made by each company will in turn affect the prices of its competitors. Thus a non-cooperative game is formed.

To study the Nash equilibrium solution of the noncooperative game, we first analyze the second derivative of $f(x)$, which is:

$$
f^{\prime \prime}(x)=\frac{A \cdot e^{x}}{\left(1+A \cdot e^{x}\right)^{3}} \cdot\left[2 A x \cdot e^{x}-(x+2)\left(1+A \cdot e^{x}\right)\right]
$$

It can be determined from the above equation that there exists an inflection point, say $x=x_{1}$, where $f^{\prime \prime}\left(x_{1}\right)=0$. $f(x)$ is a strictly concave function in the area where $x \leq x_{1}$. Several experiments have been made and we observed that $x_{1} \approx 4 x_{0}$ under different values of $A$. This means during the profit maximization process, $x$ can rarely go beyond $x_{1}$. Considering the certain issue, a new function $g(x)$ is built with the following properties:
1. $g(x)=a x^{2}+b x+c$, where $a, b, c$ are given constant values and $a<0$

2. $g\left(x_{1}\right)=f\left(x_{1}\right)$

3. $g^{\prime}\left(x_{1}\right)=f^{\prime}\left(x_{1}\right)$

And then we modify $f(x)$ to a new function $k(x)$ as follows:

$$
k(x)= \begin{cases}f(x) & \left(x \leq x_{1}\right) \\ g(x) & \left(x>x_{1}\right)\end{cases}
$$

Property 1: $k(x)$ is a strictly concave function.

Proof: $k(x)$ can be determined as continuous and differentiable due to the second and the third properties of $g(x) . f(x)$ is a strictly concave function in the area where $x \leq$ $x_{1}$, so $k^{\prime}(x)$ is strictly monotonically decreasing in this area. In addition, $g(x)$ is a well-known strictly concave quadratic function, so $k^{\prime}(x)$ is also strictly monotonically decreasing in the area $x>x_{1}$. We can therefore determine $k^{\prime}(x)$ as strictly monotonically decreasing in the whole interval of definition and thus strictly concave.

Considering the fact that $x_{1}$ is already far from the price optimization area, this kind of modification will not influence the effectiveness of the final solution.

The profit maximization problem for utility companies can be defined as follows:

$$
\begin{aligned}
\max _{\text {for all } c} & \text { profit_total }[c]=\sum_{h} k\left(\operatorname{cost}_{-} h[c][h]\right) \\
\text { s.t. } & P[c][t]>0, \quad \forall c, t \\
& P[c][t] \leq \text { max }_{-} \text {price, } \forall c, t \\
& \text { As companies are considered as non-cooperative }
\end{aligned}
$$
among each other, we are interested in the existence and uniqueness of Nash equilibrium points.

Property 2: Utility companies have a unique Nash equilibrium point in the profit maximization problem.

Proof: Note that $k(x)$ has been proved to be strictly concave, while cost_h[c][h] is an affine function of price function $P[c][t]$. We can determine that $k($ cost $h[c][h])$ is also strictly concave as a function of $P[c][t]$, and so is its summation function to $h$. Therefore, the profit maximization problem is a strictly concave $n$-person game. In this case, the existence and uniqueness of Nash equilibrium are directly resulted from the first and third theorem in [16].

Another observation is that as we consider no energy generation cost, the objective functions for different utility companies are totally symmetric.

Property 3: At the Nash equilibrium point, each utility company has exactly the same price function.

Proof: Assume the price function of two utility companies, namely $c_{1}$ and $c_{2}$, are different in a Nash 
equilibrium point (say $P\left[c_{1}\right][t] \neq P\left[c_{2}\right][t]$ ). As the objective functions of these two companies are totally the same, another Nash equilibrium point should exist if they exchange their price functions, which contradicts the uniqueness of Nash equilibrium in property 2 .

The third property makes the problem much easier to solve, as we can simply assume the price function of all utility companies are the same after the first derivative of the objective function. The detailed algorithm is presented as follows:

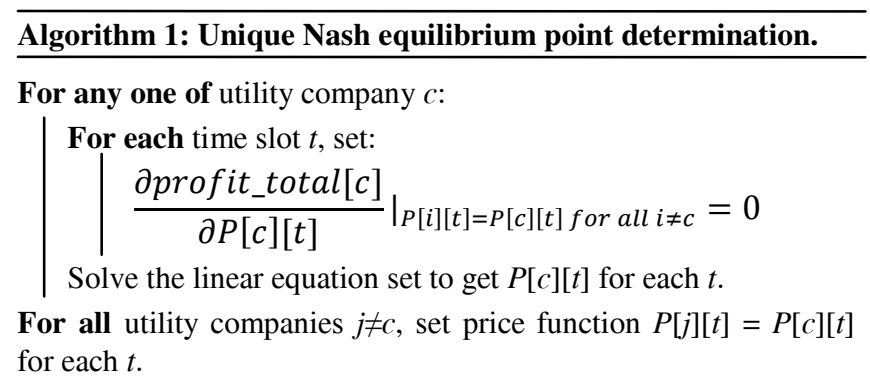

\section{MODEL II: CONSDERING ENERGY GENERATION COST}

Model I is far from complete, as utility companies should also take into consideration the cost of electricity generated by different sources, which is the cost of generating electricity at the point of connection to a load or electricity grid [1]. As the trend of the future power networks is towards distributed smart grid, the capital, maintenance and distance cost will differ significantly based on the electricity generation type (e.g., steam-power station or solar-energy-power station) as well as weather, area and seasons. In this model, we use $P_{-} e[c][t]$ to denote the energy cost function for each utility company at a certain time slot $t$. The energy generation cost for homeowner $h$ (denoted by cost_e $[c][h]$ ) can be calculated as:

$$
\text { cost_e } e[c][h]=\sum_{t} P_{-} e[c][t] \cdot \operatorname{con}[c][h][t]
$$

As has been stated in several previous papers like [11] and [17], the introduction of dynamic pricing is to regulate homeowner's energy usage at peak demand hours and thus achieve a global energy saving. Considering that homeowners will always have an incentive to move their tasks from high-price hours to low-price times. We therefore set a negative correlation between energy price and energy usage during a certain amount of time by using $P \_$expect $[h]$ to represent the expected energy price for every homeowner. The homeowner's energy cost can be reformulated as:

$$
\begin{aligned}
& \operatorname{cost}_{-} h[c][h]=\sum_{t} P[c][t] \cdot \operatorname{con}[c][h][t] \cdot\left[\frac{e}{2} \cdot\left(1+\frac{P[c][t]}{P_{-} \operatorname{expect}[h]}\right)\right. \\
& \left.\cdot e^{-\frac{P[c][t]}{P_{-} \text {expect }[h]}} \cdot(1-\alpha)+\alpha\right]
\end{aligned}
$$

where $\alpha$ is a constant value between 0 and 1 which reflects the inelastic energy consumption of each homeowner. And consequently the profit maximization problem is modified as:

$$
\begin{aligned}
& \max _{\text {for all c }} \text { profit_total }[c] \\
& =\sum_{h} \frac{\left(\cos _{-} h[c][h]-\operatorname{cost}_{-} e[c][h]\right) \cdot e^{-\operatorname{cost}_{-} h[c][h]}}{e^{- \text {cost_h }_{-}[c][h]}+\sum_{i \neq c} e^{-\operatorname{cost}_{-} h[i][h]}} \\
& \text { s. t. } P[c][t]>0, \quad \forall c, t \\
& P[c][t] \leq \text { max_price, } \quad \forall c, t
\end{aligned}
$$

Once these more sophisticated factors are taken into consideration, the properties of the first model may no longer exist. However, based on game theoretic method, this problem can be solved by iterative local maximization of each utility company. And finally we can still achieve a Nash equilibrium point under a given initial condition (i.e., the initial price function of utility companies). Notice that a constant value $d$ is needed to determine the endpoint. More precisely, the optimization process stopped when no utility companies can achieve a profit increase higher than $d$. Obviously, the value of $d$ makes a tradeoff between accuracy of the solution and run-time. The detailed algorithm is presented as follows:

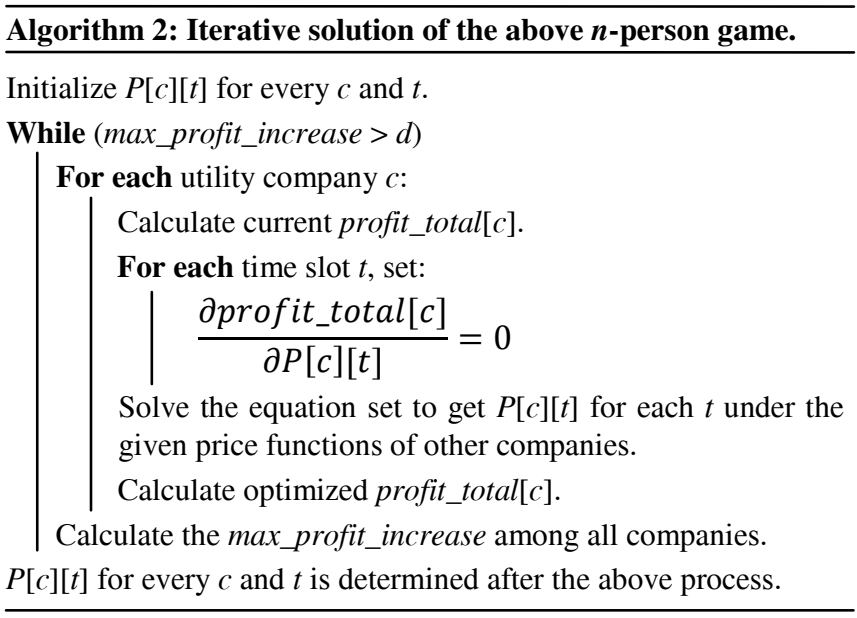

\section{Simulation Results}

To demonstrate the effectiveness of the proposed solutions, cases corresponding to the aforesaid pricing models are examined.

In these simulations, the duration of a time slot is set to one hour. For this reason, power consumption of the tasks is determined with a granularity of one hour. The proposed 
algorithms have been implemented using Matlab and tested for random cases. For both models, the energy consumption at each time slot is fixed for every homeowner, and each homeowner has a probability to choose certain utility company based on the offered price functions.

For model I, we calculated the unique Nash equilibrium point for an oligopolistic energy market containing 5 utility companies serving 100 homeowners. In order to show the effectiveness of the result, we assume an initial price function is given to all companies and they applied the calculated result one after another. Table I and Table II show the comparison between different utility companies under a high initial price and a low initial price, respectively.

Table I. Profit Comparison of model I under a high initial Price

\begin{tabular}{|c|c|c|}
\hline $\begin{array}{c}\text { Number of utility } \\
\text { companies that } \\
\text { applied the } \\
\text { calculated price }\end{array}$ & $\begin{array}{c}\text { Profit of a } \\
\text { company which } \\
\text { applied } \\
\text { calculated price }\end{array}$ & $\begin{array}{c}\text { Profit of a } \\
\text { company which } \\
\text { did not apply } \\
\text { calculated price }\end{array}$ \\
\hline 0 & - & 47.0 \\
\hline 1 & 53.9 & 33.1 \\
\hline 2 & 41.5 & 25.6 \\
\hline 3 & 33.8 & 20.9 \\
\hline 4 & 28.6 & 17.7 \\
\hline 5 & 24.7 & - \\
\hline
\end{tabular}

Table II. Profit Comparison of model I under a low initial Price

\begin{tabular}{|c|c|c|}
\hline $\begin{array}{c}\text { Number of utility } \\
\text { companies that } \\
\text { applied the } \\
\text { calculated price }\end{array}$ & $\begin{array}{c}\text { Profit of a } \\
\text { company which } \\
\text { applied } \\
\text { calculated price }\end{array}$ & $\begin{array}{c}\text { Profit of a } \\
\text { company which } \\
\text { did not apply } \\
\text { calculated price }\end{array}$ \\
\hline 0 & - & 11.8 \\
\hline 1 & 14.2 & 13.0 \\
\hline 2 & 15.9 & 14.5 \\
\hline 3 & 18.0 & 16.5 \\
\hline 4 & 20.8 & 19.1 \\
\hline 5 & 24.7 & - \\
\hline
\end{tabular}

The above tables verify that regardless of how high or low the initial price is set to, utility companies will always have incentives to adjust the price function and thus maximize their own profits. Their final price strategy turned out to be the same as the calculated result, which has been proved to be the unique Nash equilibrium solution. Note that the Nash equilibrium point does not guarantee the globally optimal solution. This can also be observed from the first table where the profit of each utility company is higher if all of them choose to retain a high energy price.
But this kind of cooperation can easily be broken since each company can achieve a profit increase by lowering its price. The result also explains why energy consumers can benefit from the price competition.

For model II, we assume different energy generation costs for utility companies and use the iterative Algorithm 2 to solve the profit maximization problem for each company. Table III is the comparison between the initial profit and final profit.

Table III. Profit Comparison of Model II for Different Utility Companies

\begin{tabular}{|c|c|c|}
\hline Company & Initial Profit & Final Profit \\
\hline 1 & 42.1485 & 27.4585 \\
\hline 2 & 40.6060 & 24.9549 \\
\hline 3 & 42.9249 & 28.6499 \\
\hline 4 & 41.0160 & 26.0111 \\
\hline 5 & 41.1806 & 26.0680 \\
\hline
\end{tabular}

Figure 1 shows the change of price function for utility companies. We assume the energy price is initially set to be high, but it is finally brought down due to competition. Companies turn out to have different final price functions due to different energy generation cost.

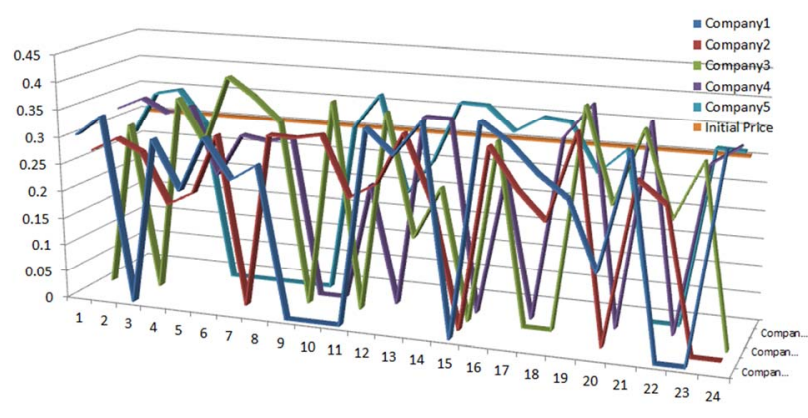

Figure 1. Initial and final price functions for each utility companies

Figure 2 illustrates the change of profit for utility companies as a factor of simulation steps. It can be observed that each company achieves a profit increase when it is running the local optimization step, while suffers from a profit decrease when other companies are running their own optimization. The curves turn out to be stable in the end, which means each company can no longer pursue profit increase by adjusting its price function. In other words, a Nash equilibrium solution is achieved. 


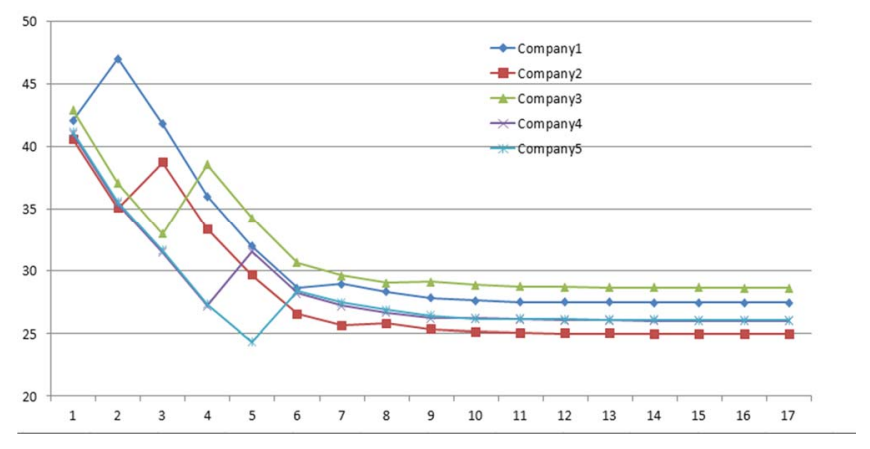

Figure 2. Profit change steps for each utility companies

Runtime of the proposed heuristic for all the 5 utility companies is about 1 second and 10 minutes for the first and second model respectively both on a machine with a dual core processor with frequency of $2.80 \mathrm{GHz}$. This run time makes it feasible to utilize our models real-time.

\section{CONCLUSION}

Two models were presented to tackle the profit maximization problem of non-cooperative utility companies in oligopolistic market. The models are under different assumptions including its problem formulation, proof of properties and optimal solution. In each model, utility companies are considered as non-cooperative, i.e., always making decisions based on their own best solution. A probability-based calculation is utilized based on consumers' reaction to price functions from different companies. The models were implemented and tested with some arbitrary test schemes. The results confirm that our designed algorithms lead to Nash equilibrium solutions for both problems, which means that all utility companies achieve their local optimal price functions. The real-time simulation strengthened the reliability of our proposed solution on price function with an acceptable runtime.

\section{REFERENCES}

[1] The Smart Grid: An Introduction. The US Department of Energy, 2008.

[2] S. Caron and G. Kesidis, "Incentive-based Energy Consumption Scheduling Algorithms for the Smart Grid," Proc. Smart Grid Communications Conf., 2010.

[3] L. Chen, S. Low, and J. Doyle, "Two market models for demand response in power networks," Proc. Smart Grid Communications Conf., 2010.
[4] Tariq Samad, "Technology Developments and R\&D Challenges for Smart Grid Applications in Homes, Buildings, and Industry, " Presentation slides.

[5] S. Hatami and M. Pedram, "Minimizing the Electricity Bill of Cooperative Users under a Quasi-Dynamic energy pricing Model," Proc. Smart Grid Communications Conf., 2010.

[6] S. Kishore, L. V. Snyder, "Control Mechanisms for Residential Electricity Demand in Smart Grids," Proc. Smart Grid Communications Conf., 2010.

[7] D. O'Neill, M. Levorato, A. Goldsmith, and U. Mitra, "Residential demand response using reinforcement learning," Proc. Smart Grid Communications Conf., 2010.

[8] H. Goudarzi, S. Hatami, and M. Pedram, "Demand-side load scheduling incentivized by dynamic energy prices," Proc. 2nd Int'l Conf. on Smart Grid Communications, Oct. 2011.

[9] http://www.powersmartpricing.org/tools

[10] P. Samadi, H. Mohsenian-Rad, R. Schober, V. Wong, and J. Jatskevich, "Optimal real-time pricing algorithm based on utility maximization for smart grid," Proc. Smart Grid Communications Conf., 2010.

[11] T. Cui, H. Goudarzi, S. Hatami, S. Nazarian, and M. Pedram, "Concurrent optimization of consumer's electrical energy bill and producer's power generation cost under a dynamic pricing model," Proc. of the 3rd IEEE PES Innovative Smart Grid Technologies Conference, Jan. 2012.

[12] N. Gregory Mankiw, "Principle of Economics," Dryden Pr, 1997.

[13] Tikuan Gu, "Thermal Power Station," China Power Press, 2008.

[14] J. Valenzuela and M. Mazumdar, "The electricity price duration curve under Bertrand and Cournot models," Proc. Conf. Probability Methods Appl. Power Syst., Ames, IA, Sep. 2004, pp. 38-43.

[15] Y. Narahari, D. Garg, R. Narayanam, H. Prakash, "Game Theoretic Problems in Network Economics and Mechanism Design Solutions," Springer, 2009, pp. 21.

[16] J. B. Rosen, "Existence and uniqueness of equilibrium points for concave n-person games," Econometrica, vol. 33, pp. 347-351, 1965.

[17] T. Cui, Y. Wang, H. Goudarzi, S. Nazarian, and M. Pedram. "Profit Maximization for Utility Companies in an Oligopolistic Energy Market with Dynamic Prices," IEEE Online Conference on Green Communications, 2012.

[18] Saad, W.; Zhu Han; Poor, H.V.; Basar, T.; , "Game-Theoretic Methods for the Smart Grid: An Overview of Microgrid Systems, Demand-Side Management, and Smart Grid Communications," Signal Processing Magazine, IEEE, vol.29, no.5, pp.86-105, Sept. 2012

[19] Mohsenian-Rad, A.-H.; Wong, V.W.S.; Jatskevich, J.; Schober, R.;, "Optimal and autonomous incentive-based energy consumption scheduling algorithm for smart grid," Innovative Smart Grid Technologies (ISGT), 2010 , vol., no., pp.1-6, 19-21 Jan. 2010 